\title{
Model-based computation of total stressed blood volume from a preload reduction manoeuvre
}

\author{
Antoine Pironet ${ }^{\mathrm{a}, 1}$, Thomas Desaive ${ }^{\mathrm{a}}$, J. Geoffrey Chase ${ }^{\mathrm{b}}$, Philippe \\ Morimont $^{\mathrm{a}}$, Pierre C. Dauby ${ }^{\mathrm{a}}$
}

${ }^{a}$ University of Liège (ULg), GIGA-Cardiovascular Sciences, Liège, Belgium

${ }^{b}$ University of Canterbury, Department of Mechanical Engineering, Christchurch, New Zealand

\begin{abstract}
Total stressed blood volume is an important parameter for both doctors and engineers. From a medical point of view, it has been associated with the success or failure of fluid therapy, a primary treatment to manage acute circulatory failure. From an engineering point of view, it dictates the cardiovascular system's behaviour in changing physiological situations. Current methods to determine this parameter involve repeated phases of circulatory arrests followed by fluid administration. In this work, a more straightforward method is developed using data from a preload reduction manoeuvre. A simple six-chamber cardiovascular system model is used and its parameters are adjusted to pig experimental data. The parameter adjustment process has three steps: (1) compute nominal values for all model parameters; (2) determine the five most sensitive parameters; and (3) adjust only these five parameters. Stressed blood volume was selected by the algorithm, which emphasizes the importance of this parameter. The model was able to track experimental trends with a maximal root mean squared error of $29.2 \%$. Computed stressed blood volume equals $486 \pm 117 \mathrm{ml}$ or 15.7 $\pm 3.6 \mathrm{ml} / \mathrm{kg}$, which matches previous independent experiments on pigs, dogs and humans. The method proposed in this work thus provides a simple way to compute total stressed blood volume from usual hemodynamic data.

Keywords: stressed blood volume, parameter identification, cardiovascular system, fluid therapy, mathematical model

\footnotetext{
${ }^{1}$ Corresponding author. Tel.: +32-4-3663356. E-mail addresses: a.pironet@ulg.ac.be (Antoine Pironet), tdesaive@ulg.ac.be (Thomas Desaive), geoff.chase@canterbury.ac.nz (J. Geoffrey Chase), ph.morimont@chu.ulg.ac.be (Philippe Morimont), pc.dauby@ulg.ac.be (Pierre C. Dauby)

Abbreviations: SBV (total stressed blood volume), ICU (intensive care unit), CVS (cardiovascular system), MCFP (mean circulatory filling pressure), CO (cardiac output), SV (stroke volume)
}

Preprint submitted to Elsevier

May 18, 2015 


\section{Introduction}

This study focuses on the computation of total stressed blood volume (SBV), a virtual volume defined as the total pressure-generating blood volume in the circulation $[3,18]$. It is an important parameter for both physicians and engineers.

From the clinician's point of view, SBV has recently regained interest in the context of fluid therapy. This therapy aims to improve cardiac output (CO) by increasing the quantity of fluid in the circulation and is frequently used to manage acute circulatory failure [19]. It has been shown to be effective only for low preload levels, leading to a distinction among patients between responders and non-responders. Consequently, clinicians have been searching for various indices of fluid responsiveness. In this context, Maas et al. have recently shown that, the lower the SBV, the higher the likelihood a patient would be a responder to fluid therapy [18].

To assist physicians, engineers have developed a wide range of mathematical models, in particular of the cardiovascular system (CVS). For these tools to be usable at an intensive care unit (ICU) bedside, they have to be fast. This requirement has led to a focus on lumped-parameter models $[6,8,24,29,31]$.

Lumped-parameter CVS models can either be open [6, 29] or closed-loop $[8,24,31]$. Open-loop models only represent a section of the CVS and thus, have input and output flows. Closed-loop models represent the whole CVS and thus, have neither input nor output flows. In other words, the total quantity of blood in such models is fixed and conserved. When using a closed-loop model, it is thus paramount to know the total blood volume in the model. More precisely, most CVS models rely on the concept of SBV.

However, this importance is often underestimated, because most studies focusing on CVS models aim at simulating situations in which the determinants of CO (inotropy, preload, afterload and heart rate [14]) are constant $[1,5,6,27,28,29,31,32,33,34]$, while the effect of SBV only appears in simulations in which these determinants change. Nevertheless, this type of simulation is the most useful, because in real life, these determinants are constantly changing due to breathing, exercise, etc., and sudden larger changes occur in dysfunctions. It is equally important clinically, where changes in state must be managed by treatment. Paradoxically, to our knowledge, few works have sought to determine this SBV value and many authors do not even mention the value used in simulation.

All previous studies to experimentally compute SBV relied on the following principle. First, the heart is stopped to let the CVS reach its equilibrium pressure, called the mean circulatory filling pressure (MCFP) [11]. Then, a given fluid volume is infused in the CVS and the heart is stopped again. The MCFP is now higher than before fluid infusion. These steps of fluid infusion and cardiac arrest can be repeated. Finally, a linear regression of the infused volume-MCFP points allows estimation of SBV as the volume that should be withdrawn for MCFP to be zero. The method is illustrated in Figure 1.

Such experiments have been performed in pigs [21] and dogs $[7,10,16,17$, 


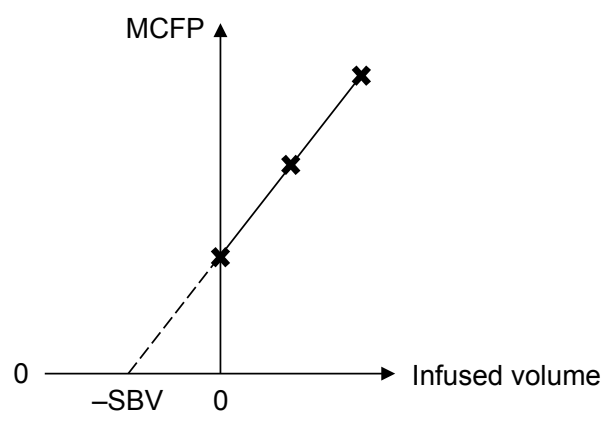

Figure 1: Illustration of the experimental method for determination of SBV.

22, 30], but are inconceivable and unethical in humans. For humans, Maas et al. [18] recently determined SBV by stopping blood flow (and measuring MCFP) in the arm instead of the whole body.

This method has many drawbacks. First, it involves repeated circulatory arrests (either global or regional). Second, fluid has to be infused, which can be harmful if the patient is a non-responder. Third, it is time-consuming (more than 20 minutes [18]). Fourth, it is highly invasive as it requires the use of at least one pressure catheter. Finally, it relies on the concept of MCFP, which is not a pressure encountered in normal situations.

This work presents a new method to estimate SBV without requiring circulatory arrests or fluid administration. This method is based on the use of a closed-loop CVS model, of which SBV is a parameter. The model is described in Section 2.1. Identification of the model parameters on preload reduction manoeuvres provides a value for SBV. This novel method is a valuable tool to track the evolution of SBV during fluid therapies.

\section{Methods}

\subsection{Cardiovascular System Model}

The CVS model used has been previously described by Smith et al. [31]. It has been validated in several animal experiments $[28,32,33,34]$. In this work, direct ventricular interaction is neglected for simplicity. The CVS model comprises six chambers representing the two ventricles ( $l v$ and $r v$ ) and the systemic and pulmonary arteries $(a o$ and $p a)$ and veins $(v c$ and $p u)$. The model chambers are joined by flow resistances representing the heart valves and the systemic and pulmonary circulations. The valvular behavior is implemented by means of the flow equivalent of a diode. The model is represented in Figure 2.

The arteries and veins are represented by purely passive chambers, where pressure $P_{i}$ and volume $V_{i}$ are linked by

$$
P_{i}(t)=E_{i}\left(V_{i}(t)-V_{U, i}\right)
$$




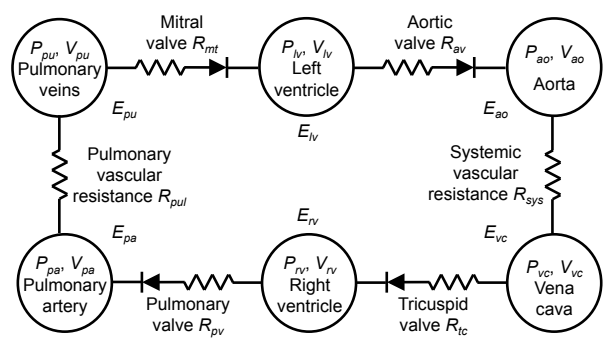

Figure 2: Cardiovascular system model.

with $i=a o, p a, v c$ and $p u$. The parameter $E_{i}$ is the elastance of the chamber. Instead of having to specify the unstressed volumes $V_{U, i}$ for all sections of the CVS taken into account, a common, simpler procedure is to perform the change of variables

$$
V_{S, i}(t)=V_{i}(t)-V_{U, i}
$$

and to work only with stressed blood volumes $V_{S, i}$. Doing so, (1) becomes

$$
P_{i}(t)=E_{i} V_{S, i}(t) .
$$

The ventricles are described by a time-varying pressure-volume relationship

$$
\begin{aligned}
& P_{l v}(t)=e_{l v}(t) E_{l v}\left(V_{l v}(t)-V_{U, l v}\right)=e_{l v}(t) E_{l v} V_{S, l v}(t), \\
& P_{r v}(t)=e_{r v}(t) E_{r v}\left(V_{r v}(t)-V_{U, r v}\right)=e_{r v}(t) E_{r v} V_{S, r v}(t)
\end{aligned}
$$

where the $E_{i}$ are the end-systolic elastances, the $V_{U, i}$ are the (constant) unstressed volumes, the $V_{S, i}$ are the stressed volumes and the $e_{i}(t)$ denote the normalized time-varying elastance functions, which can be described by [31]

$$
e_{i}(t)=\exp \left[-W_{i}\left((t \bmod T)-\frac{T}{2}\right)^{2}\right]
$$

where $W_{i}$ dictates the width of the Gaussian function and $T$ is the cardiac period. This formulation of the driver function is the simplest possible, involving only two parameters. It was chosen to make the model and the parameter identification process as simple as possible. However, such a symmetric driver function is not physiologically realistic and other models exist, but involve more parameters $[1,3,5,6,8,24]$.

As mentioned previously, the six model chambers are linked by flow resistances $R_{j}$, representing the four heart valves $(j=m t, a v, t c$ and $p v)$ and the systemic and pulmonary capillaries $\left(j=\right.$ sys and pul). Flows $Q_{s y s}$ and $Q_{p u l}$ through the systemic and pulmonary resistances $R_{\text {sys }}$ and $R_{p u l}$ are described by means of Poiseuille's equation

$$
Q_{j}(t)=\frac{\Delta P_{j}(t)}{R_{j}} \text { for } j=\text { sys and } p u l
$$


where $\Delta P_{j}$ represents the pressure difference across the resistance. In the case of the heart valves, there is flow only if the pressure difference is positive. Hence

$$
Q_{j}(t)=\left\{\begin{array}{cl}
\frac{\Delta P_{j}(t)}{R_{j}} & \text { if } \Delta P_{j}(t)>0 \\
0 & \text { otherwise }
\end{array}\right.
$$

for $j=m t, a v, t c$ and $p v$.

Finally, the rate of volume change in a model chamber $i$ is dictated by the continuity equation

$$
\dot{V}_{i}(t)=\dot{V}_{S, i}(t)=Q_{\text {in }, i}(t)-Q_{\text {out }, i}(t)
$$

where $Q_{i n, i}(t)$ and $Q_{o u t, i}(t)$ respectively represent flow going in and coming out of the chamber $i$.

Summing (9) for all six model chambers gives

$$
\sum_{i} \dot{V}_{S, i}(t)=\sum_{i} Q_{i n, i}(t)-\sum_{i} Q_{o u t, i}(t) .
$$

If the model is closed-loop, the flow coming out of a chamber is the flow going into the next, and the right-hand side cancels out. Consequently, the total stressed blood volume contained in the system is a constant

$$
\sum_{i} V_{S, i}(t)=\mathrm{SBV}
$$

The model equations are summarised in Appendix A. These equations are solved using MATLAB's ode45 solver (2010a, MathWorks, Natick, MA). Overall, the model counts 16 parameters ( 6 elastances $E_{i}, 6$ resistances $R_{j}, 2$ width parameters $W_{l v}$ and $W_{r v}$, the cardiac period $T$ and SBV). Parameter identification is used to assign values to the parameters from clinical data.

\subsection{Rationale for Identification of the Stressed Blood Volume}

This section presents the basic idea behind computation of SBV. When the CVS model is simulated in steady conditions, there is a practical indetermination between venous elastances and SBV. Indeed, SBV can be chosen completely arbitrarily if the venous elastances are adapted so that the veins store enough blood, hence resulting in the same mean venous pressures. For instance, Figure 3 shows simulated left ventricular volume, pulmonary vein pressure, left ventricular pressure and aortic pressure for the two different parameter sets given in Table 1. As shown in this table, the SBV of the second set is one third of the one from the first set. Venous elastances of the second set have been increased to compensate for this lower SBV. As can be seen in Figure 3, simulated variables are nearly identical for the two parameter sets. Consequently, venous chamber elastances and SBV cannot simultaneously be determined from measurements of left ventricular volume, pulmonary vein pressure, left ventricular pressures and aortic pressure in constant conditions. Using such data, the model is practically non-identifiable [25]. 

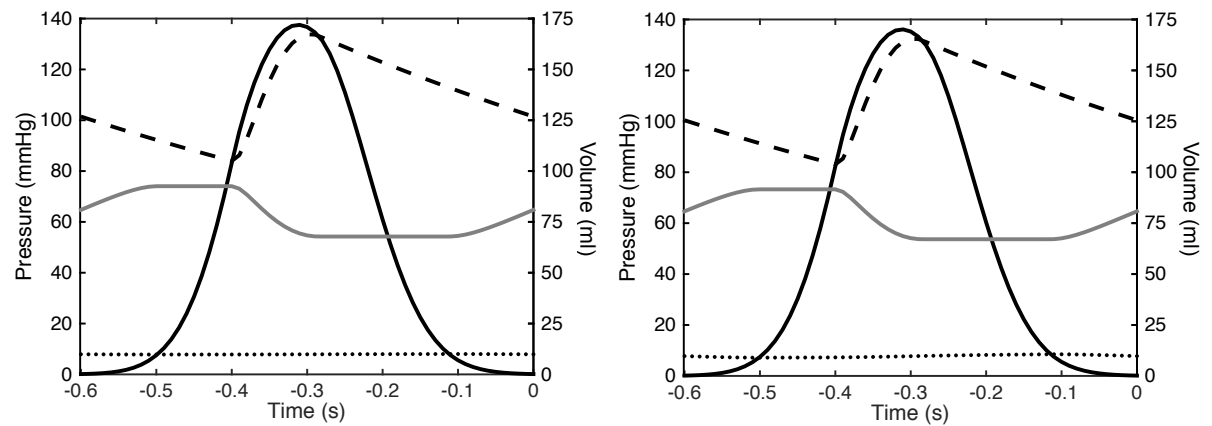

Figure 3: Simulations of the CVS model using the parameters of Table 1. Left: simulation 1, right: simulation 2. Full line: left ventricular pressure, dotted line: pulmonary vein pressure, dashed line: aortic pressure, grey line: left ventricular volume.

Table 1: Parameter values used for simulations of Figures 3 and 4.

\begin{tabular}{llcc}
\hline Parameter & Units & Simulation 1 & Simulation 2 \\
\hline$W_{l v}$ & $\mathrm{~s}^{-2}$ & 80 & 80 \\
$W_{r v}$ & $\mathrm{~s}^{-2}$ & 80 & 80 \\
$T$ & $\mathrm{~s}$ & 0.6 & 0.6 \\
$\mathrm{SBV}$ & $\mathrm{ml}$ & 1500 & 500 \\
$E_{l v}$ & $\mathrm{mmHg} / \mathrm{ml}$ & 2 & 2 \\
$E_{r v}$ & $\mathrm{mmHg} / \mathrm{ml}$ & 0.8 & 0.8 \\
$E_{a o}$ & $\mathrm{mmHg} / \mathrm{ml}$ & 2.5 & 2.5 \\
$E_{v c}$ & $\mathrm{mmHg} / \mathrm{ml}$ & 0.01 & 0.027 \\
$E_{p a}$ & $\mathrm{mmHg} / \mathrm{ml}$ & 2.1 & 2.1 \\
$E_{p u}$ & $\mathrm{mmHg} / \mathrm{ml}$ & 0.01 & 0.067 \\
$R_{s y s}$ & $\mathrm{mmHg} \mathrm{s} / \mathrm{ml}$ & 2.5 & 2.5 \\
$R_{p u l}$ & $\mathrm{mmHg} \mathrm{s} / \mathrm{ml}$ & 0.4 & 0.4 \\
$R_{m t}$ & $\mathrm{mmHg} \mathrm{s} / \mathrm{ml}$ & 0.05 & 0.05 \\
$R_{a v}$ & $\mathrm{mmHg} \mathrm{s} / \mathrm{ml}$ & 0.04 & 0.04 \\
$R_{t c}$ & $\mathrm{mmHg} \mathrm{s} / \mathrm{ml}$ & 0.04 & 0.04 \\
$R_{p v}$ & $\mathrm{mmHg} \mathrm{s} / \mathrm{ml}$ & 0.03 & 0.03 \\
\hline
\end{tabular}

However, when preload or afterload are changing, the indetermination between venous elastances and SBV vanishes. To show it, the model reaction to a preload reduction manoeuvre is simulated. The preload reduction manoeuvre is modeled as a sudden twofold increase of tricuspid valve resistance $R_{t c}$ at an instant chosen to be $t=0$. (This way of reproducing the experimental setup is discussed in Section 4.) The model reaction for the two parameter sets of Table 1 is displayed in Figure 4. As can be seen in this figure, pressures and volumes change faster in the model with a smaller SBV and larger venous elastances. This behaviour was to be expected since larger elastances mean that a given change in volume will cause larger changes in pressure. Note that the new 
steady state reached after the preload change is also different (not shown). SBV is thus important when preload is varying. This idea will be used to compute SBV from experimental data.
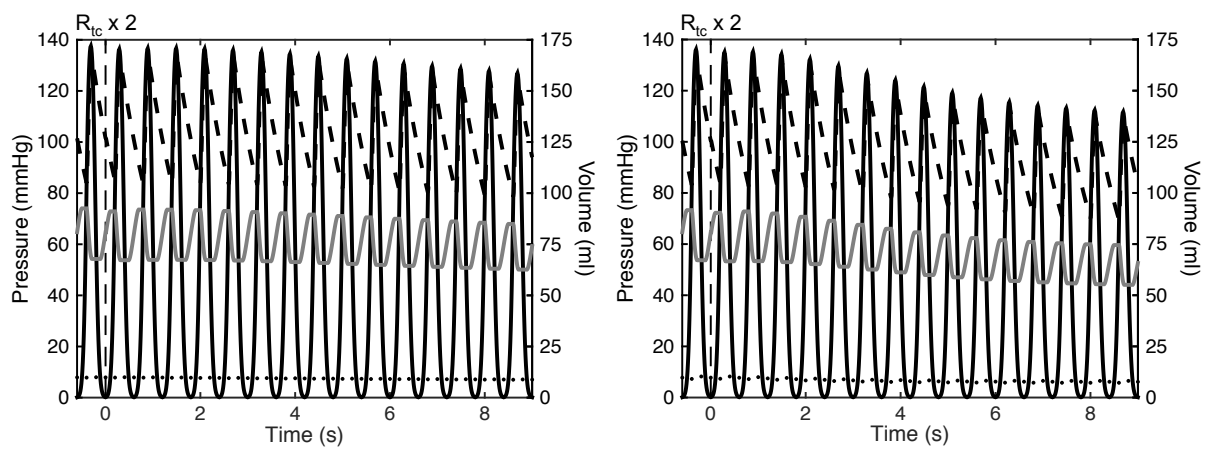

Figure 4: Simulations of the CVS model using the parameters of Table 1. Tricuspid valve resistance is doubled at $t=0 \mathrm{~s}$. Left: simulation 1, right: simulation 2. Full line: left ventricular pressure, dotted line: pulmonary vein pressure, dashed line: aortic pressure, grey line: left ventricular volume.

\subsection{Experimental data}

To identify model parameters and prove the concept, experimental animal data were used. These data came from basal state measurements on seven pigs (numbered 1 to 7 ), performed with the approval of the Ethics Committee of the Medical Faculty of the University of Liège. Details on the experimental procedures are available elsewhere $[9,15]$. Measurements comprise continuous recording of:

- left and right ventricular volumes $V_{l v}(t)$ and $V_{r v}(t)$,

- left and right ventricular pressures $P_{l v}(t)$ and $P_{r v}(t)$,

- systemic and pulmonary arterial pressures $P_{a o}(t)$ and $P_{p a}(t)$.

The pigs were also weighed at the beginning of the experiments.

A Fogarty balloon catheter was introduced in the inferior vena cava of each animal. After all sensors were correctly positioned, preload was transiently reduced by inflating this balloon. This procedure is further referred to as "preload reduction manoeuvre". In two animals (numbered 1 and 3), two preload reduction manoeuvres were performed.

In total, nine experimental datasets were thus used, relative to seven animals. Table 2 summarises the following experimental data for the nine datasets:

- mean left $\left(\bar{V}_{l v}\right)$ and right $\left(\bar{V}_{r v}\right)$ ventricular volumes,

- left $\left(\mathrm{SV}_{l v}\right)$ and right $\left(\mathrm{SV}_{r v}\right)$ ventricular stroke volumes, 
Table 2: Summary of the experimental data. The asterisk $\left(^{*}\right)$ denotes cases for which measured pulmonary artery pressure was negative at the end of the preload reduction manoeuvres.

\begin{tabular}{|c|c|c|c|c|c|c|c|c|c|}
\hline & Pig 1 & Pig 1 & Pig 2 & Pig 3 & Pig 3 & Pig 4 & Pig 5 & Pig 6 & Pig 7 \\
\hline \multicolumn{10}{|c|}{ Last heart beat before preload reduction } \\
\hline$V_{l v}(\mathrm{ml})$ & 67.4 & 64.0 & 80.2 & 66.1 & 65.3 & 56.3 & 67.1 & 63.2 & 53.7 \\
\hline $\mathrm{SV}_{l v}(\mathrm{ml})$ & 27.0 & 25.1 & 23.4 & 30.8 & 33.9 & 27.5 & 27.6 & 28.1 & 18.3 \\
\hline $\bar{P}_{a o}(\mathrm{mmHg})$ & 123.6 & 112.0 & 74.0 & 117.4 & 110.0 & 117.3 & 74.8 & 80.1 & 58.3 \\
\hline $\mathrm{PP}_{a o}(\mathrm{mmHg})$ & 30.9 & 24.7 & 19.0 & 30.4 & 26.8 & 33.2 & 34.1 & 37.1 & 21.8 \\
\hline $\bar{V}_{r v}(\mathrm{ml})$ & 55.8 & 56.7 & 62.7 & 45.2 & 45.6 & 50.5 & 66.5 & 37.9 & 49.9 \\
\hline $\mathrm{SV}_{r v}(\mathrm{ml})$ & 17.3 & 16.7 & 18.1 & 10.7 & 10.9 & 14.9 & 20.5 & 17.5 & 12.6 \\
\hline $\bar{P}_{p a}(\mathrm{mmHg})$ & 15.0 & 14.7 & 11.2 & 4.7 & 4.1 & 8.6 & 9.8 & 14.4 & 11.9 \\
\hline $\mathrm{PP}_{p a}(\mathrm{mmHg})$ & 9.6 & 9.0 & 8.6 & 7.7 & 7.3 & 9.7 & 12.0 & 12.6 & 11.6 \\
\hline \multicolumn{10}{|c|}{ Last heart beat before stopping preload reduction } \\
\hline $\bar{V}_{l v}(\mathrm{ml})$ & 47.0 & 47.1 & 63.9 & 43.1 & 49.7 & 44.9 & 50.1 & 50.5 & 50.6 \\
\hline $\mathrm{SV}_{l v}(\mathrm{ml})$ & 8.1 & 7.4 & 18.3 & 9.0 & 20.2 & 13.5 & 11.0 & 20.3 & 15.9 \\
\hline $\bar{P}_{a o}(\mathrm{mmHg})$ & 68.9 & 63.8 & 55.4 & 68.5 & 94.5 & 100.1 & 41.4 & 62.8 & 52.3 \\
\hline $\mathrm{PP}_{a o}(\mathrm{mmHg})$ & 13.8 & 10.6 & 15.7 & 16.5 & 21.2 & 26.4 & 19.4 & 31.9 & 19.4 \\
\hline $\bar{V}_{r v}(\mathrm{ml})$ & 46.3 & 46.5 & 53.9 & 42.6 & 41.9 & 41.7 & 55.8 & 33.1 & 46.4 \\
\hline $\mathrm{SV}_{r v}(\mathrm{ml})$ & 8.7 & 9.2 & 6.6 & 7.9 & 8.1 & 8.6 & 7.2 & 11.7 & 13.2 \\
\hline $\bar{P}_{p a}(\mathrm{mmHg})$ & 5.9 & 6.1 & 4.7 & $-1.8^{*}$ & $-0.9^{*}$ & $2.2^{*}$ & $0.8^{*}$ & 7.5 & 5.8 \\
\hline $\mathrm{PP}_{p a}(\mathrm{mmHg})$ & 4.8 & 4.3 & 5.5 & $7.2^{*}$ & $5.6^{*}$ & $5.7 *$ & $4.5^{*}$ & 11.5 & 6.8 \\
\hline
\end{tabular}

- mean systemic $\left(\bar{P}_{a o}\right)$ and pulmonary $\left(\bar{P}_{p a}\right)$ arterial pressures,

- systemic $\left(\mathrm{PP}_{a o}\right)$ and pulmonary $\left(\mathrm{PP}_{p a}\right)$ arterial pulse pressures.

These eight indices were computed on the last heart beat before the preload reduction manoeuvre (first eight rows of Table 2), and on the last heart beat before stopping the preload reduction manoeuvre (last eight rows).

Note that more experiments were performed to obtain data from preload reduction manoeuvres. However, the resulting measurements were not regular, due to premature ventricular contractions, and were thus not included in the present analysis. These premature contractions were probably caused by the large amount of sensors inserted in the animals' CVS.

\subsection{Parameter Identification}

The parameter identification procedure involved three steps. First, nominal values had to be assigned to all 16 model parameters. From simulations carried out using these values, an algorithm selected a sensitive subset of parameters to be further identified. Finally, this subset of parameters was identified using an iterative procedure.

\subsubsection{Nominal Parameter Values}

To assign nominal values to the model parameters, the available data was used in combination with the model equations presented in Section 2.1.

First, the cardiac period $T$ was computed by dividing the duration of the preload reduction manoeuvre by the number of cycles during the experiment. The assumption of constant cardiac period was consistent with the experimental data.

Second, $E_{l v}$ (and left ventricular unstressed volume $V_{U, l v}$ as a byproduct) have been determined by linear regression of the end-systolic pressure-volume 
points, according to the method of Kass et al. [13]. Then, the experimental driver function has been computed using (5):

$$
e_{l v}(t)=\frac{P_{l v}(t)}{E_{l v}\left(V_{l v}(t)-V_{U, l v}\right)}
$$

and the parameter $W_{l v}$ was estimated by fitting (6) to the previously computed curve. Parameters $E_{r v}, V_{U, r v}$ and $W_{r v}$ were computed by an analogous procedure.

In the previous computations, parameters $T, W_{l v}, W_{r v}, E_{l v}$ and $E_{r v}$ were computed by directly fitting the model to the data. Computation of the other parameters from the available data required some degree of approximation. When it was not possible to infer a parameter value only from the data, reference values published in the literature were used. [14]

Third, the systemic vascular resistance was computed using its definition

$$
R_{s y s}=\frac{\bar{P}_{a o}-\bar{P}_{v c}}{\mathrm{CO}}
$$

where $\bar{P}_{a o}$ and $\bar{P}_{v c}$ are, respectively, mean aortic and vena cava pressure. For simplicity, $\bar{P}_{v c}$ was neglected with respect to $\bar{P}_{a o}$ and thus set to zero in (13).

Fourth, the pulmonary vascular resistance was estimated using the pulmonary counterpart of (13)

$$
R_{p u l}=\frac{\bar{P}_{p a}-\bar{P}_{p u}}{\mathrm{CO}}
$$

where $\bar{P}_{p a}$ and $\bar{P}_{p u}$ respectively denote mean pulmonary arterial and venous pressures. Here also, $\bar{P}_{p u}$ was neglected with respect to $\bar{P}_{p a}$ and set to zero in (14).

Fifth, during diastole, volume change in the aorta is described by

$$
\dot{V}_{S, a o}(t)=-\frac{P_{a o}(t)-P_{v c}(t)}{R_{s y s}} .
$$

If, once again, $P_{v c}$ is neglected with respect to $P_{a o}$ and (3), is used, one gets

$$
\dot{V}_{S, a o}(t) \approx-\frac{E_{a o} V_{S, a o}(t)}{R_{s y s}} .
$$

Solving this equation yields

$$
V_{S, a o}(t) \approx \exp \left(-\frac{E_{a o}\left(t-t_{B D}\right)}{R_{s y s}}\right) V_{S, a o}\left(t_{B D}\right) .
$$

where $t_{B D}$ denotes the beginning of diastole. Multiplying both sides of (17) by $E_{a o}$ yields

$$
P_{a o}(t) \approx \exp \left(-\frac{E_{a o}\left(t-t_{B D}\right)}{R_{s y s}}\right) P_{a o}\left(t_{B D}\right) .
$$


Since $R_{\text {sys }}$ has been computed from (13), $E_{a o}$ can be determined by fitting the measured $P_{a o}(t)$ curve during diastole to (18). The same procedure has been applied to compute $E_{p a}$.

Sixth, valve resistances $R_{m t}, R_{a v}, R_{t c}$ and $R_{p v}$ were initialized at values provided by Revie et al. [29] in another study performed on the data used in this article, i.e.

$$
\begin{aligned}
R_{m t} & =0.05 \mathrm{mmHg} \mathrm{s} / \mathrm{ml} \\
R_{a v} & =0.04 \mathrm{mmHg} \mathrm{s} / \mathrm{ml} \\
R_{t c} & =0.04 \mathrm{mmHg} \mathrm{s} / \mathrm{ml} \\
R_{p v} & =0.03 \mathrm{mmHg} \mathrm{s} / \mathrm{ml} .
\end{aligned}
$$

Seventh, according to Zanzinger et al. [37], inferior vena cava elastance for pigs is $0.44 \mathrm{mmHg} /(\mathrm{ml} / \mathrm{kg})$. This value was divided by two to account for the two venae cavae in parallel. The nominal value of $E_{v c}$ is thus $0.22 \mathrm{mmHg} \mathrm{kg} / \mathrm{ml}$ divided by the pig's weight.

Eighth, no experimental study assessing the elastance of a pulmonary vein in pigs was found. An experimental study provided reference values for the minimum and maximum pulmonary vein pressures in pigs [2]. Using this data, the amplitude of the pulmonary vein pressure was estimated to be $9 \mathrm{mmHg}$ and $E_{p u}$ was approximated by:

$$
E_{p u}=\frac{9 \mathrm{mmHg}}{\mathrm{SV}}
$$

where SV is the stroke volume. This formula underestimates $E_{p u}$ because it assumes that all the stroke volume contributes to a pressure increase in the pulmonary veins, whereas part of it flows directly into the heart during diastole.

Finally, to evaluate SBV, experimental pressure-volume curves on dogs published by Drees and Rothe [7] were used. These pressure-volume curves are similar to the one presented in Figure 1, except that the infused volume is expressed in $\mathrm{ml} / \mathrm{kg}$. From these curves, a SBV of $31.95 \mathrm{ml} / \mathrm{kg}$ was estimated.

As mentioned before, nominal values of the parameters $T, W_{l v}, W_{r v}, E_{l v}$ and $E_{r v}$ were computed by directly fitting the model equations to the data. Consequently, it was assumed that the parameter identification process would not greatly alter these parameter values. They were thus excluded from the following selection procedure, and the remaining $1 \times 11$ parameter vector was:

$$
\mathbf{p}=\left(E_{a o} E_{p a} R_{s y s} R_{p u l} R_{m t} R_{a v} R_{t c} R_{p v} E_{v c} E_{p u} \mathrm{SBV}\right) .
$$

\subsubsection{Subset Selection Algorithm}

In this section, a subset of the parameter vector $\mathbf{p}$ is selected for optimization using a dedicated algorithm [4]. This algorithm performs a sensitivity analysis on the error vector $\mathbf{e}$ and selects the parameters to which $\mathbf{e}$ is the most sensitive.

The error vector $\mathbf{e}^{n}$ for dataset number $n$ was built as the relative error between simulated values and measured values displayed in Table 2. That is, if 
the vector $\mathbf{y}$ represents a column of Table 2 , the $k^{\text {th }}$ component of $\mathbf{e}^{n}$ is

$$
e_{k}^{n}=1-\frac{\hat{y}_{k}(\mathbf{p})}{y_{k}}
$$

where $\hat{\mathbf{y}}(\mathbf{p})$ is a vector containing the corresponding simulated values. As in Table 2, the first eight components of $\mathbf{e}^{n}$ are related to the last heart beat before the preload reduction manoeuvre, whereas the $9^{\text {th }}$ to $16^{\text {th }}$ components of $\mathbf{e}^{n}$ are similar to the previous ones, but are computed on the last heart beat before stopping the preload reduction manoeuvre. As emphasized in Table 2, in four experimental datasets, measured pulmonary artery pressure was negative at the end of the preload reduction manoeuvre. The corresponding components were not included in the error vector. Hence, $\mathbf{e}^{1}, \mathbf{e}^{2}, \mathbf{e}^{3}, \mathbf{e}^{8}$ and $\mathbf{e}^{9}$ are $1 \times 16$ vectors, while $\mathbf{e}^{4}, \mathbf{e}^{5}, \mathbf{e}^{6}$ and $\mathbf{e}^{7}$ are $1 \times 14$ vectors. Ventricular pressures were not included in the error vector since they had already been used to compute parameters $T, W_{l v}, W_{r v}, E_{l v}$ and $E_{r v}$. Finally, a global $1 \times 136$ error vector $\mathbf{e}$ was built as a concatenation of the nine error vectors $\mathbf{e}^{n}$ relative to each dataset:

$$
\mathbf{e}=\left(\mathbf{e}^{1} \mathbf{e}^{2} \mathbf{e}^{3} \mathbf{e}^{4} \mathbf{e}^{5} \mathbf{e}^{6} \mathbf{e}^{7} \mathbf{e}^{8} \mathbf{e}^{9}\right) .
$$

The subset selection algorithm used in this work was introduced by Burth et al. [4] and was used in cardiovascular modeling by Pope et al. [24]. The algorithm will be briefly described here. Further details can be found in the previous references. In summary, the input to this algorithm is the non-dimensionalized $11 \times 136$ Jacobian matrix $\mathbf{J}$, that is the derivative of the error vector with respect to the vector of model parameters $\mathbf{p}$ :

$$
[\mathbf{J}]_{k l}=\frac{\partial e_{l}}{\partial p_{k}} p_{k} .
$$

In this work, the Jacobian matrix was computed by central difference approximation using a step size of $0.1 \%$ of the parameter value.

Once this Jacobian matrix was computed, the $11 \times 11$ Hessian matrix was approximated by

$$
\mathbf{H} \approx \mathbf{J}^{\prime} \mathbf{J}^{\prime}
$$

where' denotes the transpose matrix. The hypothesis underlying this approximation is that the components of the error vector e are small [4].

The principle of the subset selection algorithm is to compute the 11 eigenvalues of $\mathbf{H}$ and to separate them in two subsets containing $\rho$ and $11-\rho$ eigenvalues. The first subset contains the $\rho$ largest eigenvalues and this $\rho$ value dictates the number of parameters that can be iteratively optimized. The value of $\rho$ was chosen as

$$
\rho=\arg \max _{i}\left(\frac{\lambda_{i}}{\lambda_{i+1}}\right) \text { with } \lambda_{i} \geq \lambda_{i+1}
$$


That is, $\rho$ was chosen so that the ratio between the two consecutive eigenvalues $\lambda_{\rho}$ and $\lambda_{\rho+1}$ was the highest. Using the previous equation, $\rho$ was found to be equal to 5 .

Let $\mathbf{V}_{\rho}$ be the $11 \times 5$ matrix containing the 5 eigenvectors of $\mathbf{H}$ associated with the 5 largest eigenvalues. To find the 5 parameters corresponding to the 5 largest eigenvalues, an $11 \times 11$ permutation matrix $\mathbf{P}$ is found through a $\mathrm{QR}$ decomposition of $\mathbf{V}_{\rho}^{\prime}$ :

$$
\mathbf{V}_{\rho}^{\prime} \mathbf{P}=\mathbf{Q} \mathbf{R} .
$$

Finally, the parameter vector $\mathbf{p}$ is rearranged using $\mathbf{P}$, which gives the 5 parameters that can be used for optimization.

In the present case, the 5 selected parameters were $E_{a o}, E_{p a}, R_{s y s}, R_{p u l}$ and $\mathrm{SBV}$. The remaining 6 parameters (namely $R_{m t}, R_{a v}, R_{t c}, R_{p v}, E_{v c}$ and $E_{p u}$ ) were kept at their nominal values.

\subsubsection{Iterative Adjustment of the Selected Parameters}

The five selected parameters were identified by an iterative procedure for each of the nine datasets. The objective of this procedure was to minimize the square of the Euclidean norm of the error vectors $\mathbf{e}^{n}$. This task was performed using the simplex method. The initial values required by this algorithm were the ones computed in Section 2.4.1. All computations were performed using MATLAB (2010a, MathWorks, Natick, MA).

\subsubsection{Sensitivity and Correlation Analyses}

In order to evaluate the sensitivity $\bar{J}_{k}$ of the error vector $\mathbf{e}$ to the $k^{\text {th }}$ parameter $p_{k}$, the Euclidean norm of each row of the Jacobian matrix was computed

$$
\bar{J}_{k}=\sqrt{\sum_{l=1}^{136}[\mathbf{J}]_{k l}^{2}} .
$$

The Hessian matrix can also be used to examine correlation between parameters [23]. To do so, the $11 \times 11$ covariance matrix $\mathbf{C}=\mathbf{H}^{-1}$ must first be computed. Then, the $11 \times 11$ correlation matrix can be obtained as

$$
[\mathbf{c}]_{m n}=\frac{[\mathbf{C}]_{m n}}{\sqrt{[\mathbf{C}]_{m m}[\mathbf{C}]_{n n}}} .
$$

The closer the element $[\mathbf{c}]_{m n}$ is to 1 (respectively -1 ), the more the parameters $p_{m}$ and $p_{n}$ are directly (respectively inversely) correlated.

\section{Results and Discussion}

\subsection{Sensitivity and Correlation Analyses}

The sensitivities $\bar{J}_{k}$ computed using (31) are displayed in Figure 5. The three parameters to which the error vector $\mathbf{e}$ is the most sensitive are SBV, 
vena cava elastance $E_{v c}$ and tricuspid valve resistance $R_{t c}$. The fact that $E_{v c}$ and $R_{t c}$ are the second and third most influent parameters is due to the way the preload reduction manoeuvre is simulated (doubling of $R_{t c}$, which is the resistance downstream of the vena cava). The fourth to seventh most influent parameters are arterial elastances $E_{a o}$ and $E_{p a}$ and vascular resistances $R_{s y s}$ and $R_{p u l}$. The three remaining valve resistances $R_{m t}, R_{a v}$ and $R_{p v}$ and pulmonary vein elastance $E_{p u}$ are the least influent parameters. This outcome emphasizes the fact that valve resistances are difficult to identify (from the data used), as already noted by Revie et al. [29].

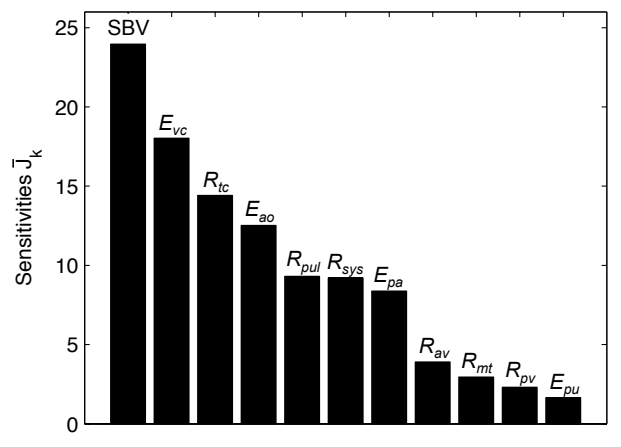

Figure 5: Sensitivities $\bar{J}_{k}$ of the error vector e to each parameter.

It should also be noted that Ellwein et al. [8] performed a parameter sensitivity analysis in a different CVS model including 11 compartments and 52 parameters. Mitral valve resistance was the $42^{\text {nd }}$ most sensitive parameter and aortic valve resistance, the $46^{\text {th }}$, which matches the present results. Note that their model did not include tricuspid and pulmonary valve resistances.

Figure 6 displays the (symmetric) correlation matrix c. The six largest (i.e. greater than 0.5 in absolute value) correlations occurred between the following parameter pairs: $E_{v c}$ and $R_{t c}, R_{a v}$ and $R_{p v}, E_{v c}$ and SBV, $E_{v c}$ and $R_{p v}, E_{p u}$ and $R_{p u l}$, and $R_{p u l}$ and $R_{m t}$.

The reason why the subset selection algorithm selected the parameters SBV, $E_{a o}, E_{p a}, R_{s y s}$ and $R_{p u l}$ can be understood by comparing Figures 5 and 6 . According to Figure 5, SBV is the parameter to which the error vector is the most sensitive. It was thus selected by the algorithm. Since SBV and $E_{v c}$ exhibit a strong correlation, $E_{v c}$ was left aside of the selected parameter set. Parameters exhibiting a strong correlation with $E_{v c}$ were also not selected $\left(R_{t c}\right.$ and $R_{p v}$ ).

The group of four parameters $E_{a o}, E_{p a}, R_{\text {sys }}$ and $R_{\text {pul }}$ exerts a moderate influence on the error vector and shows no large correlation. Consequently, it was selected by the algorithm, and parameters correlated with any of these four parameters were left aside. This was the case for $E_{p u}$ and $R_{m t}$.

The results presented in this section emphasize the importance of SBV for closed-loop CVS model simulations. The group of four parameters $E_{a o}, E_{p a}$, 


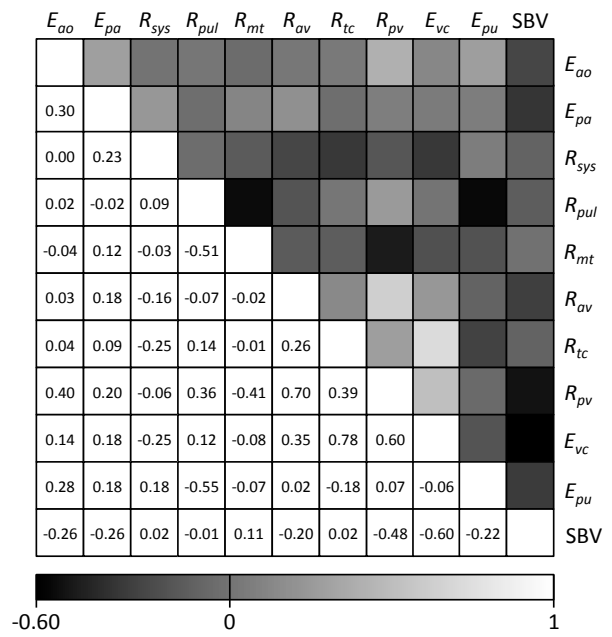

Figure 6: Correlation matrix $\mathbf{c}$ between the model parameters.

$R_{s y s}$ and $R_{p u l}$ is also shown to be relevant in a CVS model. This outcome matches and revalidates the widespread use of two-parameter windkessel models, including only an arterial elastance and a vascular resistance to represent the systemic or pulmonary arterial system.

It is worth mentioning that the sensitivity analysis and subset selection procedures were also applied to each of the nine $\mathbf{e}^{n}$ vectors separately. The individual sensitivities followed a pattern very similar to the one in Figure 5 and were also strongly correlated with the global sensitivity vector $\bar{J}_{k}$ (average $r^{2}=0.87$ ). The parameter subsets selected on the basis of the vectors $\mathbf{e}^{n}$ included the five same parameters (SBV $E_{a o}, E_{p a}, R_{s y s}$ and $R_{p u l}$ ) in $87 \%$ of the cases and rejected the same six other ones in $92 \%$ of the cases.

\subsection{Parameter Adjustment}

Table 3 shows the nominal and optimized parameter values, along with the pig weights and final values of the root mean squared error (RMSE) $\sqrt{\left\|\mathbf{e}^{n}\right\|^{2} / N_{e}}$ for all nine datasets. $N_{e}$ is the number of components in $\mathbf{e}^{n}$, equal to 14 or 16 as explained in Section 2.4.2. The largest value of the RMSE is $29.2 \%$. For the corresponding dataset, $N_{e}=14$ and not 16 because measured pulmonary artery pressure was negative at the end of the preload reduction manoeuvre. The overall poor quality of this dataset could justify this highest RMSE. Figure 7 shows simulated and measured ventricular and arterial pressures for this worstcase dataset.

For all other datasets, the RMSE lies between 18.8 and $26.2 \%$. Consequently, the parameter adjustment can be qualified as good. To further emphasize this statement, Figure 8 shows simulated and measured left ventricular and aortic pressures for pig number 7 . As can be seen on this figure, simulated and 
Table 3: Nominal and optimized values of the model parameters.

\begin{tabular}{|c|c|c|c|c|c|c|c|c|c|c|}
\hline Parameter & Units & Pig 1 & Pig 1 & Pig 2 & Pig 3 & Pig 3 & Pig 4 & Pig 5 & Pig 6 & Pig 7 \\
\hline Weight & $\mathrm{kg}$ & 35.0 & 35.0 & 31.0 & 29.0 & 29.0 & 31.0 & 32.5 & 30 & 25 \\
\hline$W_{l v}$ & $s^{-2}$ & 66.9 & 63.6 & 68.9 & 78.7 & 61.9 & 53.5 & 95.8 & 49.2 & 12.1 \\
\hline$W_{r v}$ & $\mathrm{~s}^{-2}$ & 117 & 104 & 239 & 55.1 & 53.1 & 76.0 & 238 & 76.4 & 17.1 \\
\hline$T$ & $\mathrm{~s}$ & 0.589 & 0.590 & 0.474 & 0.621 & 0.615 & 0.597 & 0.643 & 0.621 & 1.45 \\
\hline$V_{U, l v}$ & $\mathrm{ml}$ & 20.6 & 23.1 & 2.99 & 21.8 & -10.8 & -13.6 & 32.9 & -5.32 & 20.0 \\
\hline$V_{U, r v}$ & $\mathrm{ml}$ & 41.7 & 40.6 & 46.0 & 35.5 & 32.8 & 37.6 & 52.3 & 17.5 & 35.1 \\
\hline$E_{l v}$ & $\mathrm{mmHg} / \mathrm{ml}$ & 2.99 & 3.14 & 1.30 & 3.85 & 1.74 & 1.81 & 4.12 & 1.33 & 2.29 \\
\hline$E_{r v}$ & $\mathrm{mmHg} / \mathrm{ml}$ & 2.18 & 1.60 & 1.84 & 3.21 & 2.33 & 1.47 & 2.84 & 1.53 & 1.41 \\
\hline$E_{v c}$ & $\mathrm{mmHg} / \mathrm{ml}$ & 0.00629 & 0.00629 & 0.00710 & 0.00759 & 0.00759 & 0.00710 & 0.00677 & 0.00733 & 0.0088 \\
\hline$E_{p u}$ & $\mathrm{mmHg} / \mathrm{ml}$ & 0.406 & 0.431 & 0.433 & 0.434 & 0.402 & 0.425 & 0.375 & 0.395 & 0.581 \\
\hline$R_{m t}$ & $\mathrm{mmHg} \mathrm{s} / \mathrm{ml}$ & 0.0500 & 0.0500 & 0.0500 & 0.0500 & 0.0500 & 0.0500 & 0.0500 & 0.0500 & 0.0500 \\
\hline$R_{a v}$ & $\mathrm{mmHg} \mathrm{s} / \mathrm{ml}$ & 0.0400 & 0.0400 & 0.0400 & 0.0400 & 0.0400 & 0.0400 & 0.0400 & 0.0400 & 0.0400 \\
\hline$R_{t c}$ & $\mathrm{mmHg} \mathrm{s} / \mathrm{ml}$ & 0.0400 & 0.0400 & 0.0400 & 0.0400 & 0.0400 & 0.0400 & 0.0400 & 0.0400 & 0.0400 \\
\hline$R_{p v}$ & $\mathrm{mmHg} \mathrm{s} / \mathrm{ml}$ & 0.0300 & 0.0300 & 0.0300 & 0.0300 & 0.0300 & 0.0300 & 0.0300 & 0.0300 & 0.0300 \\
\hline \multicolumn{11}{|c|}{ Nominal values of the selected parameters } \\
\hline SBV & $\mathrm{ml}$ & 1120 & 1120 & 990 & 927 & 927 & 990 & 1040 & 960 & 799 \\
\hline$E_{a o}$ & $\mathrm{mmHg} / \mathrm{ml}$ & 2.19 & 2.04 & 1.03 & 2.32 & 1.97 & 2.20 & 1.61 & 2.73 & 2.25 \\
\hline$E_{p a}$ & $\mathrm{mmHg} / \mathrm{ml}$ & 0.632 & 0.726 & 0.699 & 0.682 & 0.760 & 0.775 & 0.974 & 1.44 & 1.56 \\
\hline$R_{\text {sys }}$ & $\mathrm{mmHg} / \mathrm{ml}$ & 3.29 & 3.17 & 1.69 & 3.52 & 3.02 & 3.31 & 2.00 & 2.18 & 5.45 \\
\hline$R_{p u l}$ & $\mathrm{mmHg} / \mathrm{ml}$ & 0.400 & 0.417 & 0.256 & 0.140 & 0.113 & 0.243 & 0.263 & 0.392 & 1.12 \\
\hline \multicolumn{11}{|c|}{ Optimized values of the selected parameters } \\
\hline SBV & $\mathrm{ml}$ & 535 & 536 & 436 & 535 & 498 & 567 & 342 & 669 & 256 \\
\hline$E_{a o}$ & $\mathrm{mmHg} / \mathrm{ml}$ & 1.88 & 1.59 & 2.06 & 2.28 & 2.91 & 2.88 & 2.45 & 2.61 & 1.81 \\
\hline$E_{p a}$ & $\mathrm{mmHg} / \mathrm{ml}$ & 0.860 & 0.793 & 2.74 & 2.94 & 7.07 & 9.23 & 0.960 & 2.49 & 1.11 \\
\hline$R_{s y s}$ & $\mathrm{mmHg} / \mathrm{ml}$ & 2.81 & 3.76 & 2.67 & 3.87 & 5.75 & 4.46 & 2.49 & 2.19 & 5.44 \\
\hline$R_{\text {pul }}$ & $\mathrm{mmHg} / \mathrm{ml}$ & 0.122 & 0.134 & 0.0354 & 0.00120 & 0.0115 & 0.0427 & 0.212 & 0.0846 & 0.406 \\
\hline RMSE & - & 0.212 & 0.213 & 0.262 & 0.247 & 0.292 & 0.231 & 0.211 & 0.202 & 0.188 \\
\hline$N_{e}$ & - & 16 & 16 & 16 & 14 & 14 & 14 & 14 & 16 & 16 \\
\hline SBV/Weight & $\mathrm{ml} / \mathrm{kg}$ & 15.3 & 15.3 & 14.1 & 18.4 & 17.2 & 18.3 & 10.5 & 22.3 & 10.3 \\
\hline
\end{tabular}

measured pressures are in very good agreement all along the preload reduction manoeuvre.

The errors between measurements and simulations have three main causes, which can be seen in Figures 7 and 8. First, in four datasets, measured pulmonary artery pressure is negative at the end of the experiment; these measurements are marked by asterisks in Table 2. It is uncertain whether this outcome is a measurement error or if pulmonary artery pressure was actually lower than atmospheric pressure. However, a negative pulmonary artery pressure cannot be reproduced by the CVS model, given its assumptions and formulation, which is the reason why the objective function was modified in such cases, as explained in Section 2.4.2.

Second, for all datasets, measured aortic pressure is higher than left ventricular pressure during a large part of systole. This second outcome probably comes from an error in the calibration of the pressure catheters. Simulated aortic pressure thus has to be lower than measured, because it would otherwise prevent emptying of the ventricle.

Third, measured left and right stroke volumes are different, as can be seen in Table 2. This is physiologically impossible in steady conditions (before preload reduction). Consequently, simulated stroke volume for $t<0$ must be a trade-off between measured left and right stroke volumes.

Despite these discrepancies likely due to sensor errors, the model is able to track the pressure changes when preload is reduced and the trends appear 

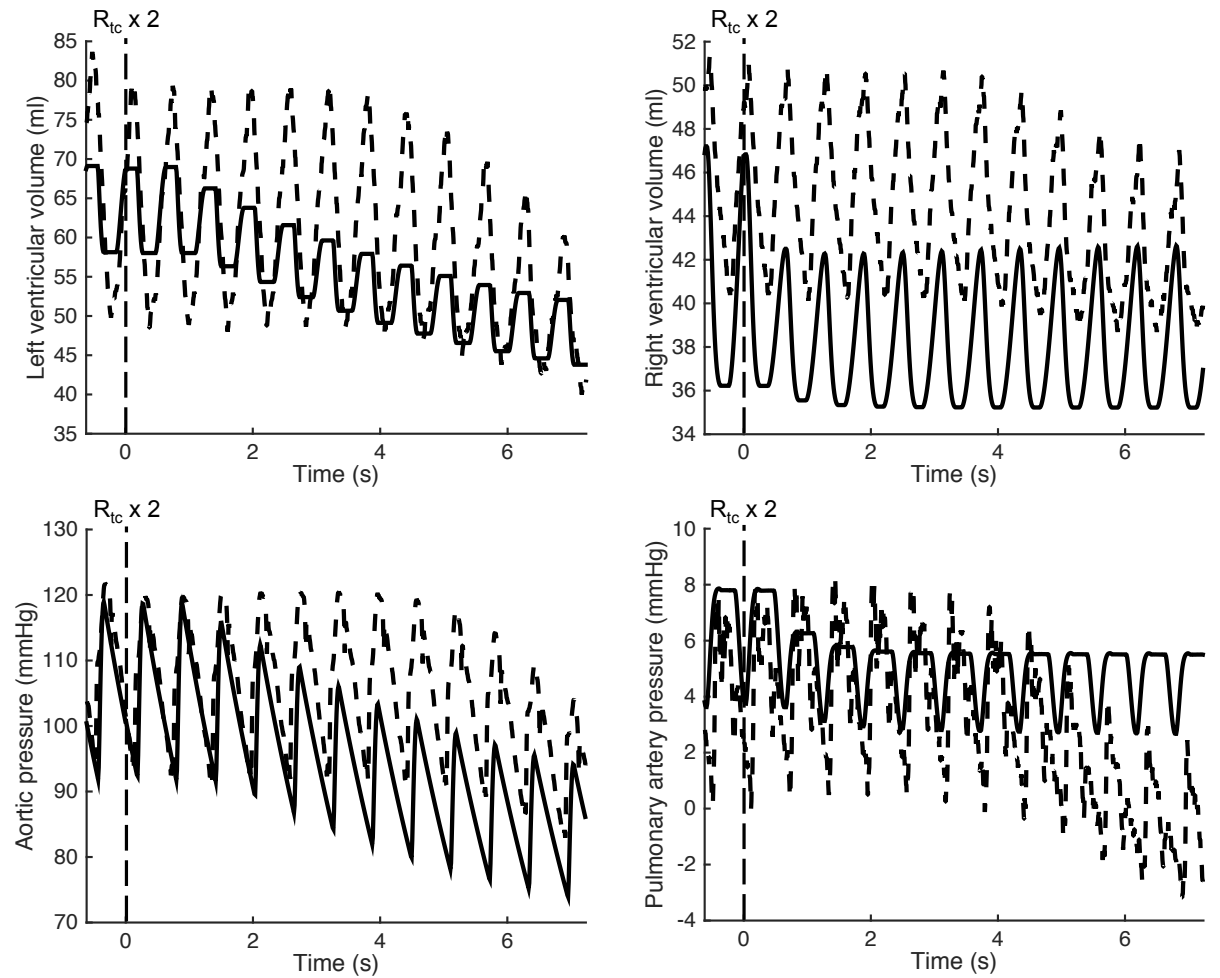

Figure 7: Result of parameter identification for pig number 3 (dataset 2). Dashed lines: measurements; full lines: simulations.

accurately reproduced, which is clinically valuable and can thus be considered as an important success. Overall, these results are the first quantitative validation of the CVS model in a situation of changing load.

\subsection{Value of the Total Stressed Blood Volume}

From the results displayed in Table 3, it can be observed that the average $\mathrm{SBV}$ for pigs is equal to $486 \pm 117 \mathrm{ml}$. For comparison, Maas et al. reported values of $1265 \pm 541 \mathrm{ml}$ in a recent study on humans [18]. There is a large intersubject variability in experimentally determined SBV values in the present study and that of Maas et al. (Coefficients of variation are 24 and $43 \%$, respectively.) This experimental study is the only one that was found providing SBV values expressed in millilitres.

The reason why no value of SBV in millilitres was found is that many investigators, when performing the experiment illustrated in Figure 1, infused a quantity of fluid expressed proportionally to the animal's weight (e.g. 10 $\mathrm{ml} / \mathrm{kg}$ ). As a consequence, these experimental studies provide a "specific" value of SBV, expressed in $\mathrm{ml} / \mathrm{kg}$. For instance, in an experimental study on pigs, 

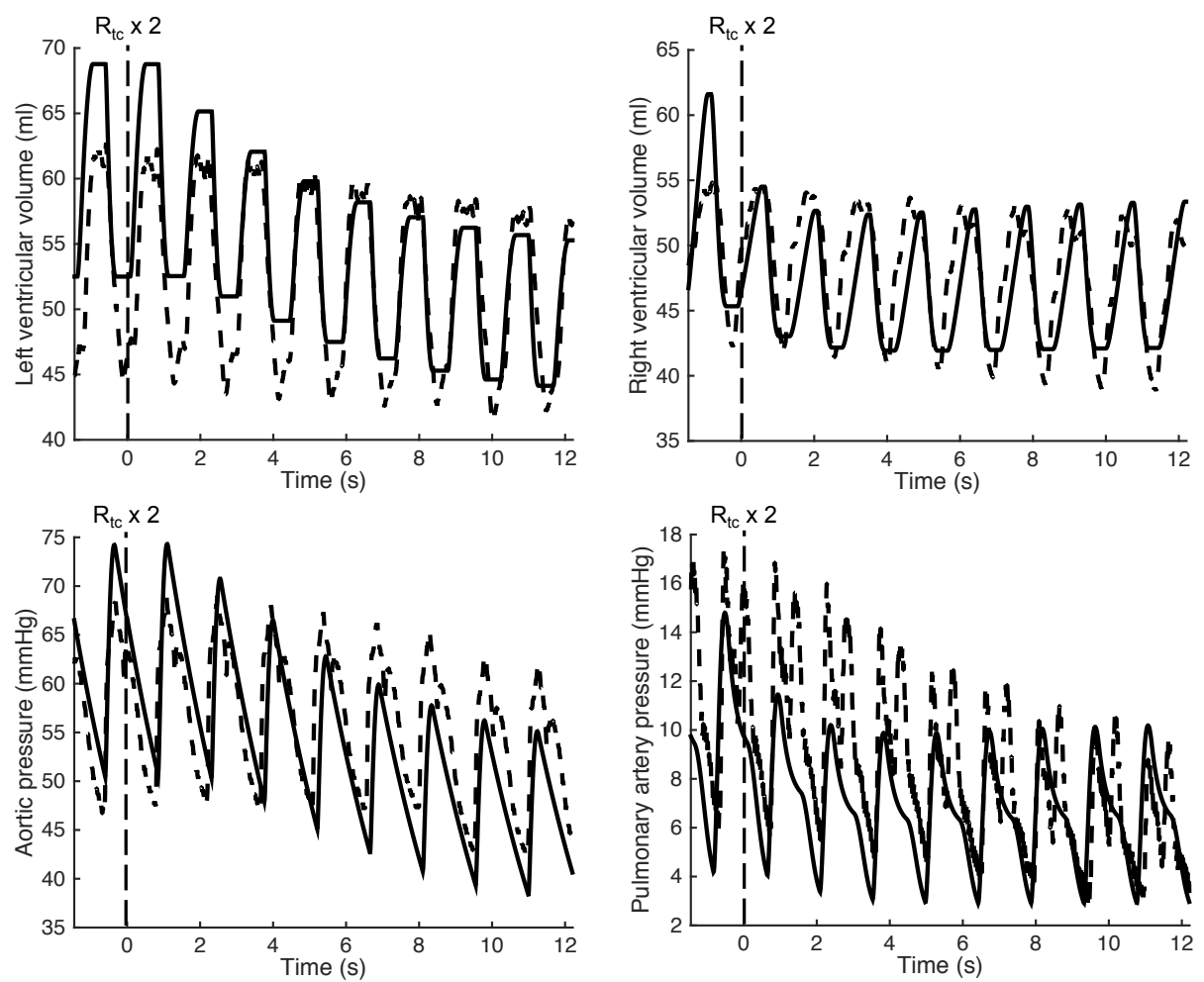

Figure 8: Result of parameter identification for pig number 7. Dashed lines: measurements; full lines: simulations.

Ogilvie et al. [21] reported mean SBV values of 29, 34 and $41 \mathrm{ml} / \mathrm{kg}$ for three different ways of inducing circulatory arrest. No other experimental study on pigs was available. In an experiment performed on dogs, Drees and Rothe [7] found values ranging from 27.8 to $43.1 \mathrm{ml} / \mathrm{kg}$, depending on the time elapsed between volume infusion or removal and circulatory arrest. The same authors [30] also reported values between 37.9 to $41.4 \mathrm{ml} / \mathrm{kg}$, depending on the amount of blood that was infused or removed. Another research group [16, 17] published curves of MCFP versus infused volume in control dogs and investigated the changes due to pharmacologic agents. In both studies, control dogs had an average SBV of $14.5 \mathrm{ml} / \mathrm{kg}$ (standard deviation was 0.2 in the first study [16], 0.5 in the second [17]).

For comparison with these "specific" values, each SBV value of Table 3 can be divided by the animal's weight (provided in the first row of Table 3). Doing so, the average "specific" SBV obtained is equal to $15.7 \pm 3.6 \mathrm{ml} / \mathrm{kg}$. The results of this study are thus of the same order of magnitude as all the previously mentioned "specific" SBV values. This observation is encouraging, but comparisons with other porcine studies would be better, if available. 
There is a large range of experimentally determined "specific" SBV values, both in this study and others. The previously reported articles have emphasized the influence of how the experiment is performed. Another possible explanation for such a large range is that there is no correlation between the weight of the pigs and the SBV value, as can be seen from Table 3. This outcome is due to the fact that SBV is not necessarily constant in a given animal. (Compare, for example, the two SBV values for pigs 1 and 3 in Table 3.) Indeed, SBV can be modified by sympathetic actions, time-dependent vascular properties, fluid exchange through the capillaries, and others [7]. Consequently, it is possibly inappropriate to define a "specific" SBV. This outcome further highlights the need to identify this subject and condition-specific parameter directly as its value in millilitres may also be clinically more relevant in titrating care.

\subsection{Other Parameter Values}

In this section, values of the parameters other than SBV are discussed. First, optimized values of the parameters are compared with their nominal values. Second, the parameter values are compared with other studies performed on the same datasets. Finally, variability of the parameters are evaluated for all datasets and for datasets coming from the same animal (pigs 1 and 3).

Maximal errors in the nominal values of parameters $E_{a o}$ and $R_{\text {sys }}$ compared with optimized values were, respectively, $52 \%$ and $36 \%$. This result justifies the formulae (13) and (18) used to compute the nominal values of these parameters and the underlying assumptions (vena cava pressure negligible with respect to aortic pressure). Interestingly, there is a mild correlation $\left(r^{2}=0.51\right)$ between nominal and optimized values of $R_{\text {sys }}$, as shown in Figure 9 (left). Using this information, a better approximation for $R_{s y s}$ is:

$$
R_{s y s} \approx 0.83 \frac{\bar{P}_{a o}}{\mathrm{CO}}+1.2 \mathrm{mmHg} \mathrm{s} / \mathrm{ml} \text {. }
$$
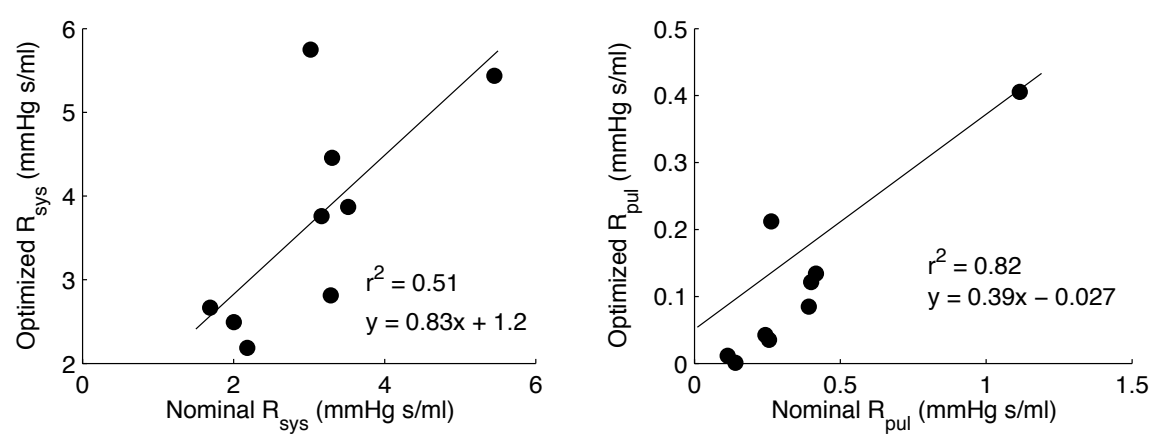

Figure 9: Linear correlation between nominal and optimized values of $R_{s y s}$ and $R_{p u l}$.

On the pulmonary side, the maximal errors in the nominal values of parameters $E_{p a}$ and $R_{p u l}$ were, respectively, 92 and $11,586 \%$. There are two 
main reasons for this large discrepancy between nominal and optimized values of $R_{\text {pul }}$. First, in the datasets, pulmonary artery pressure is particularly low, sometimes even negative, as previously mentioned. Second, in the case of the pulmonary circulation, downstream pressure cannot be neglected with respect to pulmonary artery pressure [20]. Equation (14) cannot be further simplified to $R_{p u l} \approx \bar{P}_{p a} /$ CO. However, since downstream pressure was not available, this assumption was necessary. As in the systemic case, there is a good correlation $\left(r^{2}=0.82\right)$ between nominal and optimized values of $R_{p u l}$, shown in Figure 9 (right). This result suggests the following approximation:

$$
R_{p u l} \approx 0.39 \frac{\bar{P}_{p a}}{\mathrm{CO}}-0.027 \mathrm{mmHg} \mathrm{s} / \mathrm{ml} \text {. }
$$

In other studies on the same datasets, Revie et al. [26, 27] identified all the parameters of the six-chamber CVS model except SBV. They published the values of the parameters $R_{s y s}, R_{p u l}, E_{l v}$ and $E_{r v}$. When compared to the values presented in Table 3, only the values of $R_{s y s}$ and $R_{p u l}$ are well correlated $\left(r^{2}=0.79\right.$ and 0.72 , respectively). The values of $E_{l v}$ and $E_{r v}$ show no correlation. The fact that there is no correlation between ventricular elastances comes from the fact that Revie et al. assumed $V_{U, l v}$ and $V_{U, r v}$ to be zero, which is not the case, as shown in Table 3. As explained by these authors, their values of $E_{l v}$ and $E_{r v}$ do not represent left and right ventricular contractilities in a strict sense [29].

As previously explained, two preload reduction datasets were available for pigs 1 and 3. The identified parameter values are displayed in Table 3. For the two parameter sets identified for pig 1 , the maximum parameter variabilities are approximately $30 \%$ for $E_{r v}$ and $29 \%$ for $R_{s y s}$. For all other parameters, maximum variability is $17 \%$.

For pig 3, the maximum variabilities are $591 \%$ for $V_{U, l v}$ and $162 \%$ for $R_{p u l}$. These high variabilities are caused by the fact that the pig did not react the same way to the two preload reduction manoeuvres, as shown in Figure 10. For instance, left ventricular contractility $E_{l v}$ changed by $75 \%$ between the two manoeuvres. Identified parameter values (including SBV) vary along with the changing state of the animal. This is due to the various reflex mechanisms, which are not accounted for here. It is consequently not surprising to find different parameter values for two successive measurements in a single animal. The method described in this work only aims to determine the instantaneous state of the animal.

As can be seen from Table 3, the unstressed volume $V_{U, l v}$ of the left ventricle is the most varying parameter (mean $10.2 \mathrm{ml}$ and standard deviation 16.1 $\mathrm{ml}$ ), being sometimes even negative (see for example Figure 10 (right)). This observation has been made by many other researchers [12, 36], who questioned the physiological meaning of a negative volume. The explanation of this phenomenon is that the end-systolic pressure-volume relationship is only linear on a limited volume range, but tends to become concave for low volumes [12, 36]. 

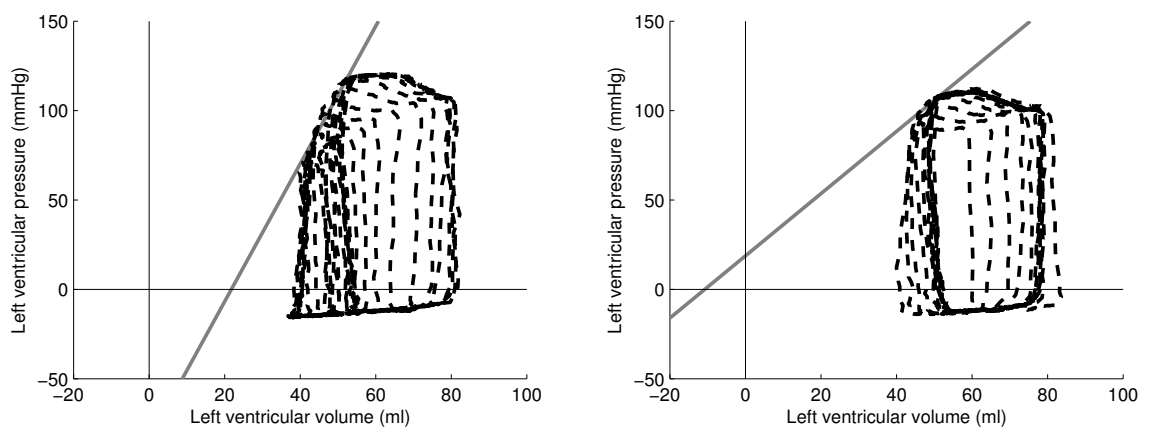

Figure 10: Left ventricular pressure-volume loops (dashed line) and end-systolic pressurevolume relationships (grey line) for the two datasets of pig 3.

\section{Limitations}

This work presented a method to compute all the parameters of a sixchamber CVS model, including total stressed blood volume, SBV, from data of preload reduction manoeuvres. In these experimental manoeuvres, one of the two input vessels to the right ventricle (the venae cavae) was occluded. In this work, it was assumed that this preload reduction manoeuvre could be simply modelled as a sharp doubling of the right ventricular input resistance $R_{t c}$. This hypothesis is discussed here.

First, to stick to the experiments, the change had to be modeled as a change in resistance (the elastance of the vessel being unaffected by the occlusion). However, the lumped-parameter nature of the model causes venae cavae to be represented as a single elastic chamber with no resistance. The remaining choice was to alter either the resistance upstream of the elastic chamber (the systemic vascular resistance $R_{\text {sys }}$ ) or the one downstream (the right ventricular input resistance $R_{t c}$ ). Since the systemic vascular resistance represents the resistance of the systemic capillaries, it seemed more appropriate to alter the right ventricular input resistance $R_{t c}$.

Second, it was assumed that the preload reduction manoeuvre could be modelled by doubling this resistance. Indeed, since one of the two input vessels is occluded, flow to the right ventricle is divided by two. Using Poiseuille's equation $Q=\Delta P / R$, if $Q$ is divided by two and $\Delta P$ remains constant, $R$ has to be doubled.

Finally, the preload reduction manoeuvre was reproduced by a sharp change of the resistance. It is likely that the computed SBV value could change according to how fast the inferior vena cava is occluded. However, measuring the time evolution of inferior vena cava occlusion requires an echographic study, which was not available here.

To validate the method presented in this work, the computed SBV value should be compared to those obtained by the experiments described in the 
introduction and in Figure 1. As mentioned previously, such experiments are invasive and risky, as they involve repetitive circulatory arrests in all or part of the CVS and multiple fluid administrations [7, 10, 16, 17, 18, 22, 21, 30]. If the method provided here correlates well with these other approaches, it could be used as a faster replacement, also avoiding the need for circulatory arrests.

Another option would be to use the presented method to track the evolution of SBV during vascular filling therapies, which is its intended use. Such experiments are currently in progress.

To overcome the present lack of experimental SBV data, the method was applied on simulated data. An example of such a numerical validation is given in Table 4. First, reference parameters were taken as 1.5 times the parameters displayed in the second column of Table 1 . Second, test data was simulated using these reference parameters. Then, nominal parameter values were obtained from the data, as described in Section 2.4.1. Table 4 shows how the formulae developed in that section perform well, especially the ones used to derive ventricular and arterial parameters. Finally, the subset selection algorithm was used and selected ten parameters for optimization. The optimized values of these parameters are also displayed in Table 4.

The SBV method was able to retrieve the parameter values used to generate the reference simulations. As expected, the most sensitive parameters such as SBV, arterial elastances and vascular resistances, were all recovered with a low relative error (below $7 \%$ ). On the other hand, the least sensitive parameters, such as valve resistances and pulmonary vein elastance are the ones the algorithm had the more difficulty to retrieve.

\section{Conclusions and Implications}

In the past, total stressed blood volume, SBV, has been considered to be an unimportant parameter of closed-loop CVS models. Its value has been omitted from numerous studies presenting such models, despite its usefulness to clinicians to assess the filling state of a patient. In this work, the importance of this parameter for CVS models was shown. Then, a simple method was presented to compute this parameter along with all other parameters of a six-chamber CVS model from usual hemodynamic data. This method consists in fitting the CVS model to data from a preload reduction manoeuvre. Because data is limited and not perfect, an algorithm was used to select a subset of parameters to fit. SBV was selected by the algorithm, which confirms the important role of this parameter. Its value could then be computed for all available datasets.

The method presented here is invasive since it is based on a preload reduction manoeuvre through vena cava occlusion. However, it could be made non-invasive by suppressing the need for ventricular pressures and volumes measurements. As previously shown, SBV is the most sensitive parameter. Therefore, it is likely to be identifiable only from aortic pressure measurements. The second step to make the method non-invasive would be to replace the vena cava occlusion by another preload reduction manoeuvre, for example a passive leg raising ma-

noeuvre (PLR) [35]. These changes would result in a fully non-invasive method 
Table 4: Numerical validation of the method. First, reference parameters were used to simulate test data. Second, nominal parameter values were obtained from this data. Third, the ten parameters selected by the subset selection algorithm were optimized.

\begin{tabular}{llrrr} 
Parameter & Units & Reference & Nominal & Optimized \\
\hline$W_{l v}$ & $\mathrm{~s}^{-2}$ & 120 & 119.9816 & \\
$W_{r v}$ & $\mathrm{~s}^{-2}$ & 120 & 119.9816 & \\
$T$ & $\mathrm{~s}$ & 0.9 & 0.9003 & \\
$\mathrm{SBV}$ & $\mathrm{ml}$ & 750 & 461.7778 & 698.2080 \\
$V_{U, l v}$ & $\mathrm{ml}$ & 0 & 0.0000 & \\
$V_{U, r v}$ & $\mathrm{ml}$ & 0 & 0.0000 & \\
$E_{l v}$ & $\mathrm{mmHg} / \mathrm{ml}$ & 3 & 3.0000 & \\
$E_{r v}$ & $\mathrm{mmHg} / \mathrm{ml}$ & 1.2 & 1.2000 & \\
$E_{a o}$ & $\mathrm{mmHg} / \mathrm{ml}$ & 3.75 & 3.7410 & 3.7406 \\
$E_{v c}$ & $\mathrm{mmHg} / \mathrm{ml}$ & 0.0405 & 0.0152 & 0.0273 \\
$E_{p a}$ & $\mathrm{mmHg} / \mathrm{ml}$ & 3.15 & 3.1040 & 3.1493 \\
$E_{p u}$ & $\mathrm{mmHg} / \mathrm{ml}$ & 0.1005 & 0.1275 & \\
$R_{s y s}$ & $\mathrm{mmHg} \mathrm{s} / \mathrm{ml}$ & 3.75 & 3.8523 & 3.8103 \\
$R_{p u l}$ & $\mathrm{mmHg} \mathrm{s} / \mathrm{ml}$ & 0.6 & 0.7330 & 0.6339 \\
$R_{m t}$ & $\mathrm{mmHg} \mathrm{s} / \mathrm{ml}$ & 0.075 & 0.0500 & 0.0581 \\
$R_{a v}$ & $\mathrm{mmHg} \mathrm{s} / \mathrm{ml}$ & 0.06 & 0.0400 & 0.0682 \\
$R_{t c}$ & $\mathrm{mmHg} \mathrm{s} / \mathrm{ml}$ & 0.06 & 0.0400 & 0.0367 \\
$R_{p v}$ & $\mathrm{mmHg} \mathrm{s} / \mathrm{ml}$ & 0.045 & 0.0300 & 0.0329 \\
\hline
\end{tabular}

to compute a patient's volume status. Non-invasive measurements are critical to enable easy identification of a patient's volume status and whether fluid therapy should be performed, which is central to monitoring and treating CVS dysfunction.

\section{Acknowledgments}

This work was supported by the French Community of Belgium (Actions de Recherches Concertées), the Belgian Funds for Scientific Research (F.R.S.FNRS) and EU Marie Curie Actions (FP7-PEOPLE-2012-IRSES).

\section{References}

[1] M. Abdolrazaghi, M. Navidbakhsh, and K. Hassani. Mathematical modelling and electrical analog equivalent of the human cardiovascular system. Cardiovascular Engineering, 10(2):45-51, 2010.

[2] P. Barbier, S. Solomon, N. B. Schiller, and S. A. Glantz. Determinants of forward pulmonary vein flow: an open pericardium pig model. Journal of the American College of Cardiology, 35(7):1947-1959, 2000. 
[3] D. Burkhoff and J. V. Tyberg. Why does pulmonary venous pressure rise after onset of lv dysfunction: a theoretical analysis. American Journal of Physiology-Heart and Circulatory Physiology, 265(5):H1819-H1828, 1993.

[4] M. Burth, G. C. Verghese, and M. Velez-Reyes. Subset selection for improved parameter estimation in on-line identification of a synchronous generator. IEEE Transactions on Power Systems, 14(1):218-225, 1999.

[5] D. C. Chung. Ventricular interaction in a closed-loop model of the canine circulation. Master's thesis, Rice University, Houston, Texas, 1996.

[6] M. Danielsen and J. T. Ottesen. Describing the pumping heart as a pressure source. Journal of Theoretical Biology, 212(1):71 - 81, 2001.

[7] J. A. Drees and C. F. Rothe. Reflex venoconstriction and capacity vessel pressure-volume relationships in dogs. Circulation Research, 34(3):360-373, 1974 .

[8] L. M. Ellwein, H. T. Tran, C. Zapata, V. Novak, and M. S. Olufsen. Sensitivity analysis and model assessment: Mathematical models for arterial blood flow and blood pressure. Cardiovascular Engineering, 8(2):94-108, 2008 .

[9] A. Ghuysen, B. Lambermont, P. Kolh, V. Tchana-Sato, D. Magis, P. Gerard, V. Mommens, N. Janssen, T. Desaive, and V. D'Orio. Alteration of right ventricular-pulmonary vascular coupling in a porcine model of progressive pressure overloading. Shock, 29(2):197-204, 2008.

[10] R. K. Goldberg, R. W. Lee, M. Olajos, and S. Goldman. Development of tolerance to nitroglycerin in the arterial and venous circulation of dogs. Journal of the American College of Cardiology, 10(6):1335-1341, 1987.

[11] A. C. Guyton, A. W. Lindsey, and B. N. Kaufmann. Effect of mean circulatory filling pressure and other peripheral circulatory factors on cardiac output. American Journal of Physiology - Legacy Content, 180(3):463-468, 1955.

[12] D. A. Kass, R. Beyar, E. Lankford, M. Heard, W. L. Maughan, and K. Sagawa. Influence of contractile state on curvilinearity of in situ endsystolic pressure-volume relations. Circulation, 79(1):167-178, 1989.

[13] D. A. Kass, W. L. Maughan, Z. M. Guo, A. Kono, K. Sunagawa, and K. Sagawa. Comparative influence of load versus inotropic states on indexes of ventricular contractility: experimental and theoretical analysis based on pressure-volume relationships. Circulation, 76(6):1422-36, 1987.

[14] R. Klabunde and A. Dalley. Cardiovascular physiology concepts. Lippincott Williams \& Wilkins, 2004. 
[15] B. Lambermont, P. Delanaye, J.-M. Dogné, A. Ghuysen, N. Janssen, B. Dubois, T. Desaive, P. Kolh, V. D'Orio, and J.-M. Krzesinski. Largepore membrane hemofiltration increases cytokine clearance and improves right ventricularvascular coupling during endotoxic shock in pigs. Artificial Organs, 30(7):560-564, 2006.

[16] R. W. Lee, R. G. Gay, L. D. Lancaster, M. Olajos, and S. Goldman. Dog model to study the effects of pharmacologic agents on the peripheral circulation: effects of milrinone. Journal of Pharmacology and Experimental Therapeutics, 240(3):1014-1019, 1987b.

[17] R. W. Lee, T. E. Raya, R. G. Gay, M. Olajos, and S. Goldman. Beta-2 adrenoceptor control of the venous circulation in intact dogs. Journal of Pharmacology and Experimental Therapeutics, 242(3):1138-1143, 1987a.

[18] J. J. Maas, M. R. Pinsky, L. P. Aarts, and J. R. Jansen. Bedside assessment of total systemic vascular compliance, stressed volume, and cardiac function curves in intensive care unit patients. Anesthesia $\mathscr{E}$ Analgesia, 115(4):880$887,2012$.

[19] F. Michard and J.-L. Teboul. Using heart-lung interactions to assess fluid responsiveness during mechanical ventilation. Critical Care, 4(5):282-289, 2000.

[20] P. Morimont, B. Lambermont, A. Ghuysen, P. Gerard, P. Kolh, P. Lancellotti, V. Tchana-Sato, T. Desaive, and V. D'Orio. Effective arterial elastance as an index of pulmonary vascular load. American Journal of Physiology - Heart and Circulatory Physiology, 294(6):H2736-H2742, 2008.

[21] R. I. Ogilvie, Zborowska-Sluis D., and Tenaschuk B. Measurement of mean circulatory filling pressure and vascular compliance in domestic pigs. American Journal of Physiology - Heart and Circulatory Physiology, 258(6):H1925-H1932, 1990.

[22] R. I. Ogilvie and D. Zborowska-Sluis. Effect of chronic rapid ventricular pacing on total vascular capacitance. Circulation, 85(4):1524-30, 1992.

[23] M. S. Olufsen and J. T. Ottesen. A practical approach to parameter estimation applied to model predicting heart rate regulation. Journal of Mathematical Biology, 67(1):39-68, 2013.

[24] S. R. Pope, L. M. Ellwein, C. L. Zapata, V. Novak, C. T. Kelley, and M. S. Olufsen. Estimation and identification of parameters in a lumped cerebrovascular model. Math Biosci Eng, 6:93-115, 2009.

[25] A. Raue, C. Kreutz, T. Maiwald, J. Bachmann, M. Schilling, U. Klingmller, and J. Timmer. Structural and practical identifiability analysis of partially observed dynamical models by exploiting the profile likelihood. Bioinformatics, 25(15):1923-1929, 2009. 
[26] J. A. Revie. Model-based cardiovascular monitoring in critical care for improved diagnosis of cardiac dysfunction. PhD thesis, University of Canterbury, 2012.

[27] J. A. Revie, D. Stevenson, J. G. Chase, C. J. Pretty, B. C. Lambermont, A. Ghuysen, P. Kolh, G. M. Shaw, and T. Desaive. Evaluation of a modelbased hemodynamic monitoring method in a porcine study of septic shock. Computational and mathematical methods in medicine, 2013, 2013.

[28] J. A. Revie, D. J. Stevenson, J. G. Chase, C. E. Hann, B. C. Lambermont, A. Ghuysen, P. Kolh, P. Morimont, G. M. Shaw, and T. Desaive. Clinical detection and monitoring of acute pulmonary embolism: proof of concept of a computer-based method. Annals of intensive care, 1(1):1-12, 2011a.

[29] J. A. Revie, D. J. Stevenson, J. G. Chase, C. E. Hann, B. C. Lambermont, A. Ghuysen, P. Kolh, G. M. Shaw, S. Heldmann, and T. Desaive. Validation of subject-specific cardiovascular system models from porcine measurements. Computer Methods and Programs in Biomedicine, 2011b.

[30] C. F. Rothe and J. A. Drees. Vascular capacitance and fluid shifts in dogs during prolonged hemorrhagic hypotension. Circulation Research, $38(5): 347-56,1976$.

[31] B. W. Smith, J. G. Chase, R. I. Nokes, G. M. Shaw, and G. Wake. Minimal haemodynamic system model including ventricular interaction and valve dynamics. Medical engineering \& physics, 26(2):131-139, 2004.

[32] B. W. Smith, J. G. Chase, G. M. Shaw, and R. I. Nokes. Experimentally verified minimal cardiovascular system model for rapid diagnostic assistance. Control engineering practice, 13(9):1183-1193, 2005.

[33] C. Starfinger, J. G. Chase, C. E. Hann, G. M. Shaw, B. Lamberrmont, A. Ghuysen, P. Kolh, P. C. Dauby, and T. Desaive. Model-based identification and diagnosis of a porcine model of induced endotoxic shock with hemofiltration. Mathematical Biosciences, 216(2):132-139, 2008.

[34] C. Starfinger, C. E. Hann, J. G. Chase, T. Desaive, A. Ghuysen, and G. M. Shaw. Model-based cardiac diagnosis of pulmonary embolism. Computer methods and programs in biomedicine, 87(1):46-60, 2007.

[35] J.-L. Teboul and X. Monnet. Prediction of volume responsiveness in critically ill patients with spontaneous breathing activity. Current Opinion in Critical Care, 14(3):334-339, 2008.

[36] N. Westerhof, N. Stergiopulos, and M. I. M. Noble. Snapshots of hemodynamics. Springer, 2010.

[37] J. Zanzinger, J. Czachurski, and H. Seller. Role of calcium-dependent $\mathrm{K}^{+}$ channels in the regulation of arterial and venous tone by nitric oxide in pigs. Pflügers Archiv, 432(4):671-677, 1996. 


\section{Appendix A. Summary of the Cardiovascular System Model Equa- tions}

Pressure-volume relationships of the aorta, vena cava, pulmonary artery and pulmonary veins:

$$
\begin{aligned}
& P_{a o}(t)=E_{a o} V_{S, a o}(t) \\
& P_{v c}(t)=E_{v c} V_{S, v c}(t) \\
& P_{p a}(t)=E_{p a} V_{S, p a}(t) \\
& P_{p u}(t)=E_{p u} V_{S, p u}(t) .
\end{aligned}
$$

Time-varying pressure-volume relationship of the left and right ventricles:

$$
\begin{aligned}
& P_{l v}(t)=e_{l v}(t) E_{l v} V_{S, l v}(t) \\
& P_{r v}(t)=e_{r v}(t) E_{r v} V_{S, r v}(t) .
\end{aligned}
$$

Time-varying elastance functions:

$$
\begin{aligned}
& e_{l v}(t)=\exp \left[-W_{l v}\left((t \bmod T)-\frac{T}{2}\right)^{2}\right] \\
& e_{r v}(t)=\exp \left[-W_{r v}\left((t \bmod T)-\frac{T}{2}\right)^{2}\right] .
\end{aligned}
$$

Flows through the systemic and pulmonary resistances:

$$
\begin{aligned}
Q_{s y s}(t) & =\frac{P_{a o}(t)-P_{v c}(t)}{R_{s y s}} \\
Q_{p u l}(t) & =\frac{P_{p a}(t)-P_{p u}(t)}{R_{p u l}} .
\end{aligned}
$$

Flows through the mitral, aortic, tricuspid and pulmonary valves:

$$
\begin{aligned}
Q_{m t}(t) & =\left\{\begin{array}{cl}
\frac{P_{p u}(t)-P_{l v}(t)}{R_{m t}} & \text { if } P_{p u}(t)>P_{l v}(t) \\
0 & \text { otherwise }
\end{array}\right. \\
Q_{a v}(t) & =\left\{\begin{array}{cl}
\frac{P_{l v}(t)-P_{a o}(t)}{R_{a v}} & \text { if } P_{l v}(t)>P_{a o}(t) \\
0 & \text { otherwise }
\end{array}\right. \\
Q_{t c}(t) & =\left\{\begin{array}{cl}
\frac{P_{v c}(t)-P_{r v}(t)}{R_{t c}} & \text { if } P_{v c}(t)>P_{r v}(t) \\
0 & \text { otherwise }
\end{array}\right. \\
Q_{p v}(t) & =\left\{\begin{array}{cl}
\frac{P_{r v}(t)-P_{p o}(t)}{R_{p v}} & \text { if } P_{r v}(t)>P_{p a}(t) . \\
0 & \text { otherwise }
\end{array}\right.
\end{aligned}
$$

Rate of volume change in the chambers:

$$
\begin{aligned}
& \dot{V}_{S, a o}(t)=Q_{a v}(t)-Q_{s y s}(t) \\
& \dot{V}_{S, v c}(t)=Q_{s y s}(t)-Q_{t c}(t)
\end{aligned}
$$




$$
\begin{aligned}
& \dot{V}_{S, p a}(t)=Q_{p v}(t)-Q_{p u l}(t) \\
& \dot{V}_{S, p u}(t)=Q_{p u l}(t)-Q_{m t}(t) \\
& \dot{V}_{S, l v}(t)=Q_{m t}(t)-Q_{a v}(t) \\
& \dot{V}_{S, r v}(t)=Q_{t c}(t)-Q_{p v}(t) .
\end{aligned}
$$

The initial conditions for solving Equations (A.15) to (A.20) are arbitrary, except that they have to verify the following equation (conservation of total stressed blood volume):

$$
V_{S, a o}(0)+V_{S, v c}(0)+V_{S, p a}(0)+V_{S, p u}(0)+V_{S, l v}(0)+V_{S, r v}(0)=\mathrm{SBV} .(\mathrm{A} .21)
$$

